\title{
Selection of the Rotation Angle Law of the Special Vehicles' Wheels
}

\author{
Alexey Germanovich Amosov \\ Assistant, Department of Engineering Graphics \\ Moscow Aviation Institute (National Research University) \\ Russia, Moscow \\ lamosov@yandex.ru
}

\begin{abstract}
The process of delivery of indivisible goods is carried out by special transport. These units are a two-link road train and consist of a tractor and semitrailer (the total length of the road train is more than $35 \mathrm{~m}$ ). Typically, the power and coupling properties of the tractor are not enough to meet the requirements for cross-country ability, so the semitrailer is equipped with an additional power plant and 4-8 pairs of active wheels.

Conventional two-link road trains combine the positive qualities of two vehicles at once - a towing vehicle and a trailer. If these vehicles are all-wheel drive, then these vehicles turn into articulated road trains with all driving (active) wheels and doubled payload, capable of delivering off-road bulky indivisible goods.
\end{abstract}

Keywords: rotation angle, law, rotation, special transport, cargo delivery, active semi-trailer, active axis, rotary axis.

\section{INTRODUCTION}

During the movement of any wheeled unit between the wheels and the roadway, significant loads arise in the contact patch, especially when cornering, affecting the trajectory of movement, and therefore it is very important to correctly evaluate the parameters of the steering system [1 - 3]. In the case of special vehicles, this issue is more acute due to the significant mass of the road train (16 tons per axle load). Next, we will consider the distribution of the rotation angles of the wheels of the semi-trailer.

The kinematic relationship between the steering angles of the left and right steered wheels when turning the car has been the subject of many works by various researchers, and some statistical material has been accumulated [2, 4 9]. Therefore, upon further consideration, we will assume that the law of distribution of rotation angles between the left and right wheels is known. It is necessary to determine the relationship between the steering angles of the steered wheels along the axles of the semi-trailer, considering the angles average between the steering angles of the left and right wheels. The scheme of accepted notation is given in Fig. 1. 


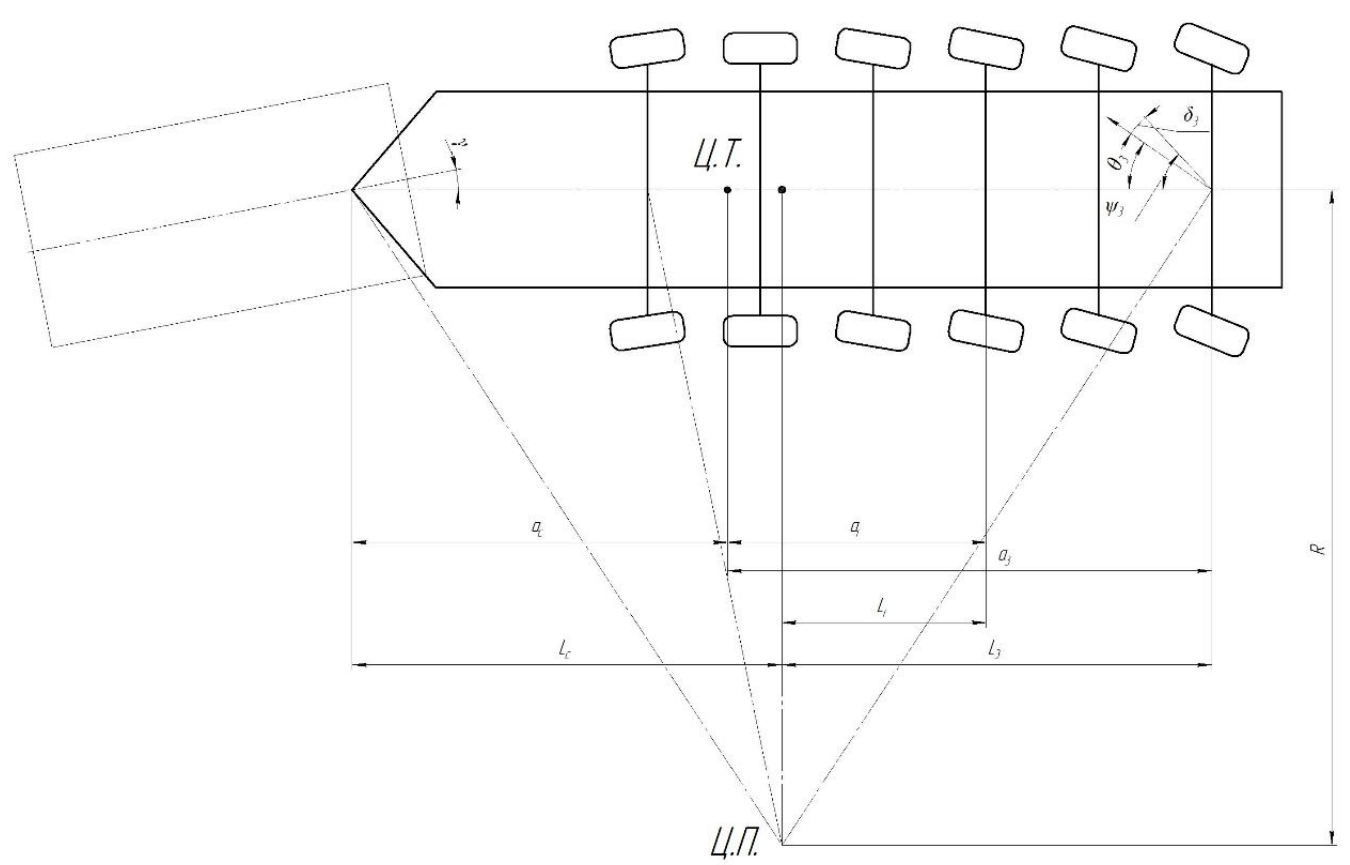

Figure 1: The scheme of accepted designations

$a_{\mathrm{i}}$ is the distance from the projection of the center of gravity on the longitudinal axis of the semi-trailer to the i-th axis; $\Omega_{\mathrm{i}}$ is the distance from the projection of the instantaneous center of rotation on the longitudinal axis to the i-th axis; $\Pi_{\mathrm{c}}$ is the distance from the projection of the instantaneous center of rotation on the longitudinal axis to the hitch; $\theta_{3}$ is the angle of rotation of the wheels of the last (rear) axis; $\psi_{3}$ is the angle between the velocity vector of the center of the rear axle and the longitudinal axis of the semitrailer; $\delta_{3}$ is the angle of the rear axle; $\mathrm{R}$ is the distance from the instantaneous center of rotation to the axis of the semi-trailer; $\gamma$ is the folding angle of the road train.

The direction in which the axis velocity vector deviates when exposed to centrifugal force is taken as the positive direction of the angles. Therefore, the angle $\delta_{3}$ is always positive, the angle $\theta_{1}$ is always negative.

Due to the fact that multi-axle high-cross-country trains are usually designed so that normal axle loads are assigned approximately the same, all further considerations will come from this assumption.

\section{METHODOLOGY}

The law of change in the angle of rotation of the steered wheels of the first, second, third, etc. axles, compared with the angle of rotation of the steered wheels of the last (rear) axis, can be different depending on the type and purpose of the unit. It can be selected on the basis of conditions for ensuring uniform wear of tire wheels of various axles, or from the condition of ensuring maximum turning moment, etc.

We will further consider the following cases of choosing the law of the ratio of rotation angles of the steered wheels of the rear and i-th axles:

a) based on the conditions for ensuring the linear law of the distribution of angles of withdrawal along the axes;

b) based on the conditions of equality of wear of tire wheels of the steered axles;

c) based on the conditions for ensuring the maximum turning moment created by the wheels of the steered axles.
We derive the equation of the ratio of the angles of rotation of the wheels of the last and i-th axes with the linear law of the distribution of the angles of withdrawal along the controlled axes of the semi-trailer. To do this, we write the equation of the distance from the projection of the center of rotation on the longitudinal axis of the semitrailer to the i-th axis:

$$
\Omega_{i} \approx-R \psi_{i}
$$

assuming that $\operatorname{tg} \psi_{\mathrm{i}}=\psi_{\mathrm{i}}$. Then from the equation $\psi_{\mathrm{i}}=\theta_{\mathrm{i}}$ $+\delta_{\text {i }}$ we determine

$$
\theta_{i}=-\frac{\Omega_{i}}{R}-\delta_{i}
$$

Since the conclusion is made under the assumption of a linear law of the distribution of angles of withdrawal along the axes, then

$$
\delta_{i}=\delta_{3} \frac{\alpha_{i}}{a_{1}}
$$

Consequently,

$$
\begin{gathered}
\theta_{i}=-\frac{\pi_{i}}{R}-\delta_{3} \frac{\alpha_{i}}{a_{3}}(1) \\
\theta_{3}=-\frac{\pi_{1}}{R}-\delta_{3}(2)
\end{gathered}
$$

Divide (1) by (2):

$$
\frac{\theta_{i}}{\theta_{1}}=\frac{\frac{\pi_{i}}{R}+\delta_{3} \frac{\alpha_{i}}{a_{3}}}{\frac{J_{3}}{R}+\delta_{3}}(3)
$$

Considering equations (1), (2) and (3), it can be noted that, with the linear law of the distribution of the angles of withdrawal along the axes, the ratios of rotation angles depend on the geometric arrangement of the axes on the longitudinal axis of the semi-trailer and the position of the 
center of gravity of the unit. In this case, the angle of deviation of the $\mathrm{i}$-th axis will be noticeably smaller than the angle of deviation of the rear axis $\delta_{3}$. A number of researchers argue that tire wear is in a quadratic dependence on the amount of angle of withdrawal, so the i-axis will be put in more favorable conditions from the point of view of wear than, for example, the rear axle, which is irrational. In addition, if the axial drag coefficients are equal, the lateral force acting on the axis of number $\mathrm{i}$ will be less. This will create a smaller turning point than one would have, which will undoubtedly affect the value of the minimum turning radius.

As a result, the selected law of the ratio of the angles of rotation of the steered wheels of the i-th axis is not sufficient. The wheels of the i-th axle under this law do not reach the intended angle of rotation, the quality of the semi-trailer in understeer is not fully used, the wear of the tires of the steered wheels is uneven.

We derive another equation for determining the ratio of the angles of rotation of the steered wheels of the i-th axis, based on the condition of equality of the angles of removal of the steered axles, i.e., the uniformity of wear of the steered wheels of all axles.

$$
\text { If } \delta_{\mathrm{i}}=\delta_{3} \text {, then } \theta_{i}=-\frac{\pi_{i}}{R}-\delta_{3}
$$

Consequently,

$$
\frac{\theta_{i}}{\theta_{3}}=\frac{\frac{\pi_{i}}{R}+\delta_{3}}{\frac{\pi_{3}}{R}+\delta_{3}} \approx \frac{\pi_{i}}{\pi_{3}}(4)
$$

In this case, the ratio of the steering angle of the steered wheels depends only on the geometric arrangement of the axles on the longitudinal axis of the semi-trailer.

With a rigid kinematic drive, the specified ratio of the steering angle of the steered wheels can be satisfied only for certain driving conditions, for other driving conditions it will not be optimal.

Formulas (3) and (4) give slightly different results, since the value $\delta_{3}$ has a small value in comparison with the other terms of the equation; therefore, formula (4), although it is insufficient, can still be used in practical calculations.

We further analyze the last case of the ratio of rotation angles of the controlled axes, based on the conditions for using the maximum turning moment.

In order to obtain a greater turning moment (for example, to obtain smaller turning radii or to exit from a deep gauge), the rotation angles of the steered wheels of all axles must be increased. Undoubtedly, it is unlikely that it will be possible to make the maximum angle of rotation of the steered wheels of the i-th axle larger than that of the rear steered axle for structural reasons. This is due to the fact that the angle of rotation of the rear steered axis is usually made as possible as possible, based on the layout of the body or frame, or on the basis of the design capabilities of the cardan joint of the wheel drive. Therefore, in the zone of maximum values of the angle of rotation of the rear steered wheels, we define the following equation for the dependence of the rotation angles of the ith controlled axis on the rotation angle of the front controlled axis:

$$
\theta_{\mathrm{i}}=\theta_{3}
$$

Under these conditions, the angles of removal of the controlled axes will not be the same. Define the values of the angles of withdrawal, which will then occur on the i-th axis:

$$
\begin{aligned}
& \text { Since } \psi_{i}=\theta_{i}+\delta_{i}=\theta_{3}+\delta_{i} \text {, then } \\
& \quad \delta_{i}=-\frac{J_{i}}{R}-\theta_{3}=-\frac{J_{i}}{R}+\frac{J_{3}}{R}+\delta_{3}=\frac{\pi_{3}-J_{i}}{R}+\delta_{3}
\end{aligned}
$$

The average range of steering angles is transitional from one law to another. Therefore, the ratio of the rotation angles $\theta_{3}$ and $\theta_{\mathrm{i}}$ must satisfy the inequality.

$$
\frac{\frac{\pi_{i}}{R}+\delta_{1} \frac{\alpha_{i}}{a_{1}}}{\frac{\pi_{1}}{R}+\delta_{1}} \leq \frac{\theta_{i}}{\theta_{3}}<1 \text { (6) }
$$

Summing up, we can say that the law of change in the angle of rotation of the steered wheels of any of the axes cannot be the same for all units regardless of its purpose. The law of change of rotation angles for wheeled vehicles, which require high transport qualities, as well as high cross-country ability and maneuverability should provide both the maximum turning force in the range of large values of the angle of rotation of the wheels and uniform tire wear on all steered wheels in the range of small values of the angle of rotation of the steered wheels.

\section{RESULT}

The question of the relationship between the folding angle of the road train and the angle of rotation of the rear axle remains unclear. If we approach the choice of rotation angles of the rear steered axis without taking into account the features of road trains with steered wheels, then we can obtain an aggregate with a clearly expressed instability of movement. The steered wheels do not affect the dynamic stability of the stationary movement of the road train, but the deterioration in the stability of the rectilinear movement with steered wheels is observed. This is due to the stabilization of the steered wheels. During unsteady motion on steered wheels, the direction of the reactive force acting in the contact print can change. As indicated in the theory of stability of motion of biaxial vehicles, under any position of the kingpin it is fundamentally impossible to achieve that the stabilizing moment on the rear steered wheels remains positive for all angles of rotation of the wheels. Since the steered wheels of the semi-trailer are used only as an auxiliary turning mechanism, it is necessary to ensure that the lateral reaction in the contact of the tires of the steered wheels does not change its direction, then there will be no deterioration in the stability of movement due to stabilization of the steered wheels. In this case, the use of steered wheels as an auxiliary turning mechanism is understood to mean such a mode of movement when a steering angle is created on the steered wheels so that the wheels do not create a turning moment, but only remove the moment of resistance to rotation that they could create if they moved in uncontrolled wheel mode. The steering system on steered wheels must be designed so that the wheels roll either without a pull or even with some positive pull, creating little resistance to turning. In order for the steered wheels to never go into a mode of movement with a negative pull and to not change the direction of the acting reactive force, it is necessary to set the following ratio of the folding angle and the angle of the last (rear) steered axis:

$$
\theta_{3}=\frac{\pi_{3}}{\pi_{c}}(\gamma+0,1)(7)
$$


Considering that the angle $\theta \mathrm{z}$ is always positive, to ensure the stability of the movement, it is desirable to have no turning of the rear steered wheels up to $\gamma \approx 5^{\circ}$ (from the experience of designing the special transport). With a further increase in the folding angle of the road train, the amount of rotation of the rear steered wheels should be determined from equation (7). For high-traffic trains when driving with very small turning radii and low speeds, i.e. in the zone of maximum values of the folding angle, where it is required to create the maximum turning moment, it must be borne in mind that from the layout condition $\theta_{\ni} \leq 1$ $\theta \mid$. Where $|\theta|$ the maximum possible angle of rotation of the rear axle.

Thus, the choice of the angle of rotation of the rear steered axis must be assigned based on the following inequality:

$$
\frac{\pi_{3}}{\pi_{\mathrm{c}}}(\gamma+0,1) \leq \theta \ni \leq|\theta|
$$

\section{CONCLUSIONS}

1. The ratio of the angles of rotation of the steered wheels of various axles should depend on the type and purpose of the unit.

2. For transport units in which durability is the most important quality, the ratio of the rotation angles of the steered wheels of different axles should ensure minimal tire wear, and, therefore, be determined by the formula (3) or (4).

3. For units of special purpose, in which crosscountry ability and overcoming obstacles are no less important qualities compared to durability, the law of changing the ratio of the steering angles of the steered wheels, the various axles should provide maximum torque at the maximum steering angles of the front steered wheels, and also ensure equality wear at slight angles of rotation of the front wheels. The law of change in the ratio of the angle of rotation of the steered wheels in the entire range of their changes should be determined in this case by inequality (6).

4. To ensure the stability of movement of multi-axle vehicles, the law of the ratio of the folding angle of the road train and the rear steered wheels should be determined by inequality (8).

\section{REFERENCES}

[1] G.I.Gladov, A.M. Petrenko. Special vehicles: Theory. Moscow: IKC "Akademkniga", 215 p, 2006.

[2] Y. Kh. Zakin. Maneuverability of the car and the road train. Moscow: Transport, 136 p, 1986.

[3] B.N.Belousov, S.D. Popov. Extra-heavy wheeled vehicles. Design. Theory. Payment. Moscow: Publishing House of MSTU named after N.E. Bauman, 728 p, 2006.

[4] N.L.Ivanina, V.I. Golovchenko. Automated calculation and construction of the overall lane of long-base truck trains when they are rotated $90^{\circ}$ and $180^{\circ}$. News of NTU "KhPI". No. 1 (975). Pp. 48 64, 2013

[5] V. A.Gorelov, S. L. Tropin. A mathematical model of the curved motion of a road train on an undeformable support base. Journal of Automotive Engineers. No. 5 (70). Pp. 18 - 22, 2011.

[6] V.F.Grekov,S.V.Orlov, A.A.Pyankov, Yu.A. Tkachenko. The influence of the structural layout of vehicles on their maneuverability. Information Processing Systems. No. 3 (70). Pp. 34 - 38, 2008.

[7] D.I.Naradovy, B.M.Pozin, I.P. Troyanovskaya. On the problem of stationary rotation of road trains. Bulletin of the South Ural State University. Series "Engineering". No. 14, issue 7. Pp. 97 - 99. 2005.

[8] S.Cheng, D. Cebon. Improving roll stability of articulated heavy vehicle using active semi-trailer steering. Vehicle System Dynamics: International Journal of Vehicle Mechanics and Mobility, Vol 46, Supplement: 373-388, 2008. https://doi.org/10.1080/00423110801958576

[9] C.Fletcher, C.Manzieand M.Good. Trailer Steering: An Australian Research Perspective and Application By-Wire Control. Technical report, Ninth International Symposium on Heavy Vehicle Weights and Dimensions. June 18-22, Penn State, State College, Pennsylvania, 2006. 